\title{
THE MAXIMALLY SYMMETRIC SURFACES IN THE 3-TORUS
}

\author{
SHENG BAI, VANESSA ROBINS, CHAO WANG, AND SHICHENG WANG
}

\begin{abstract}
Suppose an orientation-preserving action of a finite group $G$ on the closed surface $\Sigma_{g}$ of genus $g>1$ extends over the 3 -torus $T^{3}$ for some embedding $\Sigma_{g} \subset T^{3}$. Then $|G| \leq 12(g-1)$, and this upper bound $12(g-1)$ can be achieved for $g=n^{2}+1,3 n^{2}+1,2 n^{3}+1,4 n^{3}+$ $1,8 n^{3}+1, n \in \mathbb{Z}_{+}$. The surfaces in $T^{3}$ realizing a maximal symmetry can be either unknotted or knotted. Similar problems in the non-orientable category are also discussed. The connection with minimal surfaces in $T^{3}$ is addressed and the situation when the maximally symmetric surfaces above can be realized by minimal surfaces is identified.
\end{abstract}

\section{Contents}

1. Introduction

2. Upper and lower bounds of extendable finite groups 4

3. Unknotted examples of the maximally symmetric surfaces 6

4. Knotted examples of the maximally symmetric surfaces $\quad 10$

5. Minimal surfaces, space groups, proof of the main result $\quad 15$

References

\section{INTRODUCTION}

Let $\Sigma_{g}$ be the closed orientable surface of genus $g>1, \Pi_{g}$ be the closed non-orientable surface of genus $g>2$, and $T^{3}$ be the three dimensional torus (3-torus for short). We consider the following question in the smooth category:

Suppose the action of a finite group $G$ on a closed surface $S$ can extend over $T^{3}$. Then what is the maximum order of the group and what does the maximal action look like?

A similar problem has been addressed for surfaces embedded in the 3sphere, $S^{3}$, which is the simplest compact 3 -manifold in the sense that it is a one point compactification of three space and the universal spherical 3-manifold covering all spherical 3-manifolds. See [WWZZ1], [WWZZ2] for orientation-preserving actions, and [WWZ] in the general case. Note that

2010 Mathematics Subject Classification. 57M60, 57N10, 57S25, 53A10, 20F65, 05C10;

Key words and phrases. maximal surface symmetry in 3-torus, minimal surface.

The second author is supported by grant No.11501534 of NSFC and the last author is supported by grant No.11371034 of NSFC. 
only orientable surfaces $\Sigma_{g}$ can be embedded in $S^{3}$. $T^{3}$ is another natural and significant 3-manifold for this question. It is the universal compact Euclidean 3-manifold in the sense that it covers all compact Euclidean 3-manifolds; moreover $T^{3}$ is covered by 3 -space (3-dimensional Euclidean space) and the preimage of a closed surface $S \subset T^{3}$ under such a covering can be a triply periodic surface, which is an interesting object in the natural sciences and engineering [HBLL+].

Definition 1.1. Let $G$ be a finite group. A $G$-action on a closed surface $S$ is extendable over $T^{3}$ with respect to an embedding $e: S \hookrightarrow T^{3}$ if $G$ can also act on $T^{3}$ such that $h_{T^{3}} \circ e=e \circ h_{S}$ for any $h \in G$.

For an embedding of an orientable surface in $T^{3}$ we can define whether it is unknotted in the same way as the usual definition for knotting in $S^{3}$.

Definition 1.2. An embedding $e: \Sigma_{g} \hookrightarrow T^{3}$ is unknotted if $e\left(\Sigma_{g}\right)$ splits $T^{3}$ into two handlebodies. Otherwise it is knotted.

We assume all orientable manifolds in this note are already oriented. According to whether the action preserves the orientation of the surface and $T^{3}$, we can define four classes of maximum orders.

Definition 1.3. Let $S$ be either $\Sigma_{g}$ or $\Pi_{g}$. Define $E(S), E^{+}(S), E_{+}\left(\Sigma_{g}\right)$ and $E_{+}^{+}\left(\Sigma_{g}\right)$ as below:

$E(S)$ : the maximum order of all extendable $G$-actions on $S$.

$E^{+}(S)$ : the maximum order of all extendable $G$-actions on $S$ which preserve the orientation of $T^{3}$.

$E_{+}\left(\Sigma_{g}\right)$ : the maximum order of all extendable $G$-actions on $\Sigma_{g}$ which preserve the orientation of $\Sigma_{g}$.

$E_{+}^{+}\left(\Sigma_{g}\right)$ : the maximum order of extendable $G$-actions on $\Sigma_{g}$, which preserve the orientations of both $T^{3}$ and $\Sigma_{g}$.

We will prove the following Theorem 1.4 and Theorem 1.5. Theorem 1.4 gives the upper bounds of those invariants defined in Definition 1.3, and Theorem 1.5 provides infinitely many $g$ for which the upper bound is realized.

Theorem 1.4. Suppose the genus $g>1$ for $\Sigma_{g}$ and $g>2$ for $\Pi_{g}$.

(1) $E_{+}^{+}\left(\Sigma_{g}\right) \leq 12(g-1)$.

(2) $E_{+}\left(\Sigma_{g}\right) \leq 24(g-1), E^{+}\left(\Sigma_{g}\right) \leq 24(g-1), E\left(\Sigma_{g}\right) \leq 48(g-1)$.

(3) $E^{+}\left(\Pi_{g}\right) \leq 12(g-2), E\left(\Pi_{g}\right) \leq 24(g-2)$.

If an extendable $G$-action on $S$ realizes one of the above upper bounds, then the corresponding surface $e(S)$ can be thought as a maximally symmetric surface in $T^{3}$.

Theorem 1.5. Suppose $n$ is any positive integer.

(1) The upper bound of $E_{+}^{+}\left(\Sigma_{g}\right)$ in Theorem 1.4 can be achieved by an unknotted embedding for $g=2 n^{3}+1,4 n^{3}+1,8 n^{3}+1$; and by a knotted 
embedding for $g=2 n^{3}+1,4 n^{3}+1,8 n^{3}+1$, where 6 does not divide $n$; and $g=n^{2}+1,3 n^{2}+1$.

(2) The upper bound of $E_{+}\left(\Sigma_{g}\right), E^{+}\left(\Sigma_{g}\right)$ and $E\left(\Sigma_{g}\right)$ in Theorem 1.4 can be achieved for $g=2 n^{3}+1,4 n^{3}+1,8 n^{3}+1$ by an unknotted embedding.

(3) The upper bound of $E^{+}\left(\Pi_{g}\right)$ and $E\left(\Pi_{g}\right)$ in Theorem 1.4 can be achieved for $g=2 n^{3}+2,8 n^{3}+2$, where $n$ is odd.

The proof of Theorem 1.4, using the Riemann-Hurwitz Formula and the Equivariant Loop Theorem, is given in $\S 2$. The proof of Theorem 1.5 occupies the remaining three sections of the paper, which is outlined below:

In $\S 3$ and $\S 4$ we will construct various examples to realize the upper bounds in Theorem 1.5 (1), $\S 3$ for the unknotted case and $\S 4$ for the knotted case, therefore Theorem 1.5 (1) follows, up to the verification of the knottiness of those embeddings. Those examples are explicit in the following sense: The 3-torus is obtained by standard opposite face identification of the cube and we define those surfaces in the cube before the identification. Indeed, for each $g$ in Theorem 1.5 (1), we construct all embeddings $\Sigma_{g} \subset T^{3}$ realizing $E_{+}^{+}\left(\Sigma_{g}\right)$ we can image for the moment, and we expect those are all embeddings realizing $E_{+}^{+}\left(\Sigma_{g}\right)$, see a conjecture below. The constructions in $\S 3$ and $\S 4$ often rely on an understanding of crystallographic space groups.

The knottedness of the examples in $\S 3$ and $\S 4$ involved in Theorem 1.5 (1), as well as Theorem 1.5 (2) and (3), could be verified by arguments such as those in $\S 3$ and $\S 4$, and by some topological reasoning; but we present a more convenient and interesting way in $\S 5$. In $\S 5$ we first link the verification of knottedness of the examples in $\S 3$ and $\S 4$ with minimal surfaces in $T^{3}$, or equivalently, triply periodic minimal surfaces in $R^{3}$, which itself is an important topic, see $[\mathrm{Me}]$ and $[\mathrm{FH}]$ for examples. Historically, many minimal surfaces in $T^{3}$ were constructed using the symmetry of $T^{3}$ and it is known that minimal surfaces in $T^{3}$ must be unknotted [Me]. We identify the examples in $\S 3$ with known triply periodic minimal surfaces (up to isotopy) $[\mathrm{Br}]$, therefore they are unknotted. On the other hand, by a simple criterion from covering space theory and our constructions, the examples in $\S 4$ are knotted, therefore can not be realized by minimal surfaces. We also identify the group actions in $\S 3$ and $\S 4$ with known space groups as well as their index-2 supergroups; then Theorem 1.5 (2) and (3) are proved.

Corollary 1.6. $\Sigma_{g} \subset T^{3}$ achieving the upper bound $E_{+}^{+}(g)=12(g-1)$ can be realized by minimal surfaces for $g=2 n^{3}+1,4 n^{3}+1,8 n^{3}+1$.

We also show in Example 2.3 that for each $g$ it is easy to construct an extendable action of order $4(g-1)$ on $\left(T^{3}, \Sigma_{g}\right)$.

We end the introduction by the following

Conjecture 1.7. Suppose the genus $g>1$. 
(1) All $g$ realizing the upper bound $E_{+}^{+}\left(\Sigma_{g}\right)=12(g-1)$ are listed in Theorem 1.5 (1), and moreover, all examples realizing those $g$ are listed in $\S 3$ and $\S 4$.

(2) $E_{+}^{+}\left(\Sigma_{g}\right)=4(g-1)$ for $g \in Z_{+} \backslash K$, where $K=\left\{f_{i}(n) \mid n \in Z_{+}\right\}$and $f_{i}$ runs over finitely many quadratic and cubic functions.

\section{UPPER AND LOWER BOUNDS OF EXTENDABLE FINITE GROUPS}

We need the following two important results, see $[\mathrm{Hu}],[\mathrm{MY}]$, to prove Theorem 1.4.

Riemann-Hurwitz Formula. $\Sigma_{g} \rightarrow \Sigma_{g^{\prime}}$ is a regular branched covering with transformation group $G$. Let $a_{1}, a_{2}, \cdots, a_{k}$ be the branched points in $\Sigma_{g^{\prime}}$ having indices $q_{1} \leq q_{2} \leq \cdots \leq q_{k}$. Then

$$
2-2 g=|G|\left(2-2 g^{\prime}-\sum_{i=1}^{k}\left(1-\frac{1}{q_{i}}\right)\right)
$$

Equivariant Loop Theorem. Let $M$ be a three manifold with a smooth action of a discrete group $G$. Let $F$ be an equivariant subsurface of $\partial M$. If $F$ is not $\pi_{1}$-injective with respect to inclusion into $M$, then it admits a G-equivariant compression disk.

Here a nonempty subset $X$ of $M$ is $G$-equivariant if $h(X)=X$ or $h(X) \cap$ $X=\emptyset$, for any $h \in G$. A disk $D \subset M$ is a compression disk of $F$ if $\partial D \subset F$ and in $F$ it does not bound any disk.

Proof of (1). Suppose there is an extendable $G$-action on $\Sigma_{g}$ which preserves the orientations of both $T^{3}$ and $\Sigma_{g}$. Cutting $T^{3}$ along $e\left(\Sigma_{g}\right)$ we get a three manifold $M$. Since both $\Sigma_{g}$ and $T^{3}$ are orientable, $\Sigma_{g}$ must be two-sided in $T^{3}$, therefore $M$ contains two boundaries $F_{1}$ and $F_{2}$, and $F_{1} \cong F_{2} \cong \Sigma_{g}$.

Now clearly $G$ acts on $M$. Since the $G$-action preserves the orientations of both $T^{3}$ and $\Sigma_{g}$, each of $F_{1}$ and $F_{2}$ is a $G$-equivariant surface. Since $g>1$, $\pi_{1}\left(\Sigma_{g}\right)$ is not abelian, but $\pi_{1}\left(T^{3}\right)$ is abelian, the induced homomorphism $\pi_{1}\left(\Sigma_{g}\right) \rightarrow \pi_{1}\left(T^{3}\right)$ is not injective. Then at least one of $F_{1}$ and $F_{2}$ is not $\pi_{1}$-injective with respect to the inclusion into $M$. Suppose $F_{1}$ is not $\pi_{1^{-}}$ injective, then it admits a $G$-equivariant compression disk $D$ of $F_{1}$ by the Equivariant Loop Theorem.

If there exists an $h \in G$ such that $h(D)=D$ and $h$ reverses an orientation of $D$, then we can choose a $G$-equivariant regular neighbourhood $N(D)$ of $D$ such that there is a homeomorphism $i: D \times[-1,1] \rightarrow N(D)$ and $i(D \times\{0\})=D$. Then $D^{\prime}=i(D \times\{1\})$ is also a $G$-equivariant compression disk of $F_{1}$, and clearly for this $D^{\prime}$, if $h^{\prime}\left(D^{\prime}\right)=D^{\prime}$ for some $h^{\prime} \in G$, then $h^{\prime}$ preserves an orientation of $D^{\prime}$. Hence we can assume each element of $G$ that preserves $D$ also preserves its orientation, in particular it is fixed point free on $\partial D$.

Since the $G$-action on $T^{3}$ preserves the orientations of both $\Sigma_{g}$ and $T^{3}$, the induced $G$-action on $M$ preserves $F_{1}$. With the quotient topology $F_{1} / G$ 
is homeomorphic to some $\Sigma_{g^{\prime}}$, and $p: F_{1} \rightarrow F_{1} / G$ is a regular branched covering. Since the $G$ action is fixed point free on $\partial D$ by previous discussion, $p(\partial D)$ is a simple closed curve in $F_{1} / G$.

Let $a_{1}, a_{2}, \cdots, a_{k}$ be the branch points having indices $q_{1} \leq q_{2} \leq \cdots \leq q_{k}$. Note $2-2 g<0$. By the Riemann-Hurwitz Formula we have

$$
2-2 g^{\prime}-\sum_{i=1}^{k}\left(1-\frac{1}{q_{i}}\right)=\frac{2-2 g}{|G|}<0 .
$$

If $g^{\prime}=0$ and $k \leq 3$, then $p(\partial D)$ must bound a disk in $F_{1} / G$ containing at most one branch point, and $\partial D$ will bound a disk in $F_{1}$, a contradiction. Hence either $g^{\prime} \geq 1$ or $g^{\prime}=0$ and $k \geq 4$.

Notice that for each $q_{i}$, we have

$$
1-\frac{1}{q_{i}} \geq 1 / 2
$$

Then by elementary calculation we have $|G| \leq 12(g-1)$, and moreover the equality holds if and only if $g^{\prime}=0, k=4$ and $\left(q_{1}, q_{2}, q_{3}, q_{4}\right)=(2,2,2,3)$.

Remark 2.1. Zimmermann first proved that the order of an orientationpreserving finite group action on a handlebody of genus $g$ is bounded by $12(g-1)[\mathrm{Zi}]$ soon after the work [MY]. As mentioned by one of the referees, May first discovered the sharp 12(g-1) upper bound for the automorphism group of a genus- $g$ Klein surface (i.e. the case when $\mathrm{S}$ is a non-orientable surfaces, or has non-empty boundary) [Ma1]. It would be interesting to compare the maximal groups from this paper with those in the May [Ma2] and Zimmermann papers.

By the above proof, we actually have the following:

Theorem 2.2. Suppose $\Sigma_{g}$ is embedded in a three manifold $M$ and a finite group $G$ acts on $\left(\Sigma_{g}, M\right)$. If $G$ preserves both sides and the orientation of $\Sigma_{g}$ and $\Sigma_{g}$ is not $\pi_{1}$-injective in $M$, then $|G| \leq 12(g-1)$.

Now (2) and (3) follow from (1).

Proof of (2). Suppose there is an extendable $G$-action on $\Sigma_{g}$. Let $G^{o}$ be the normal subgroup of $G$ containing all elements that preserve both the orientations of $\Sigma_{g}$ and $T^{3}$. In the case of $E_{+}\left(\Sigma_{g}\right)$ or $E^{+}\left(\Sigma_{g}\right)$, the index of $G^{o}$ in $G$ is at most two, and in the case of $E\left(\Sigma_{g}\right)$, the index of $G^{o}$ in $G$ is at most four. Hence $E_{+}\left(\Sigma_{g}\right) \leq 24(g-1), E^{+}\left(\Sigma_{g}\right) \leq 24(g-1)$, $E\left(\Sigma_{g}\right) \leq 48(g-1)$.

Proof of (3). Suppose there is an extendable $G$-action on $\Pi_{g}$ and the action preserves the orientation of $T^{3}$. We can choose an equivariant regular neighbourhood $N\left(\Pi_{g}\right)$ of $\Pi_{g}$, then $G$ also acts on $\partial N\left(\Pi_{g}\right)$. Since $T^{3}$ is orientable, $N\left(\Pi_{g}\right)$ is homeomorphic to a twisted [-1,1]-bundle over $\Pi_{g}$ and $\partial N\left(\Pi_{g}\right)$ is the orientable double cover of $\Pi_{g}$ under the bundle projection. Since $\chi\left(\Pi_{g}\right)=2-g, \chi\left(\partial N\left(\Pi_{g}\right)\right)=4-2 g=2-2(g-1)$. It 
follows that $\partial N\left(\Pi_{g}\right)$ is homeomorphic to $\Sigma_{g-1}$. Clearly the $G$-action preserves the two sides of $\partial N\left(\Pi_{g}\right)$. Since the action preserves the orientation of $T^{3}$, it also preserves the orientation of $\partial N\left(\Pi_{g}\right)$. Then by the result of (1), we have $E^{+}\left(\Pi_{g}\right) \leq 12(g-2)$. And similar to the proof of (2), we have $E\left(\Pi_{g}\right) \leq 24(g-2)$.

Example 2.3. Let $\left([0,1]^{3},+\right)$ be the unit cube with a cross properly embedded shown as the right side of Figure 1. For each $g>1$, put $g-1$ copies of $\left([0,1]^{3},+\right)$ to get $\left([0,1]^{3},+\right)_{g-1}$ which is a cuboid with properly embedded antennae, as shown in Figure 1. If we identify the opposite faces of the cuboid, we get $\left(T^{3}, \Theta_{g}\right)$, where $\Theta_{g}$ is a graph of genus $g$. If we first only identify the right and left faces, we get $\left([0,1]^{2} \times S^{1}, A_{g-1}\right)$, where $A_{g-1}$ is a circular antennae with $g-1$ bars. It is easy to see that $G=D_{g-1} \oplus Z_{2}$ acts on the pair $\left([0,1]^{2} \times S^{1}, A_{g-1}\right)$, where $D_{g-1}$ is the dihedral group acts in a standard way on $\left([0,1]^{2} \times S^{1}, A_{g-1}\right)$, and $Z_{2}$ is $\pi$-rotation of $\left([0,1]^{2} \times S^{1}, A_{g-1}\right)$ around the center-circle. Clearly this action preserves each pair of faces of $\left([0,1]^{2} \times S^{1}, A_{g-1}\right)$ to be identified, therefore induces an action on $\left(T^{3}, \Theta_{g}\right)$. Let $N\left(\Theta_{g}\right)$ be a $G$-invariant regular neighborhood of $\Theta_{g}$. Then $\partial N\left(\Theta_{g}\right)=\Sigma_{g}$ and $G$ of order $4(g-1)$ acts on $\left(T^{3}, \Sigma_{g}\right)$.

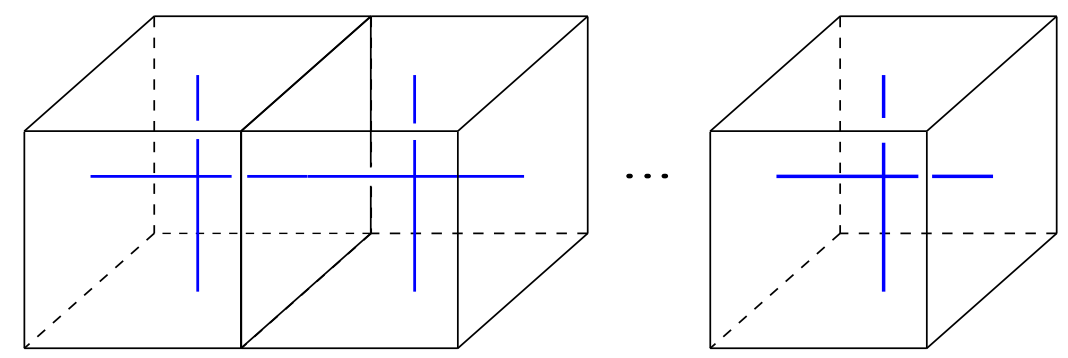

Figure 1. Cuboid with embedded antennae

\section{UNKNOTTED EXAMPLES OF THE MAXIMALLY SYMMETRIC SURFACES}

In this section we give three classes of examples realizing the upper bound of $E_{+}^{+}\left(\Sigma_{g}\right)=12(g-1)$. In each case we first construct a triply periodic graph $\Gamma$ in the three-dimensional Euclidean space $E^{3}$. There will be a three-dimensional space group $\mathcal{G}$ preserving $\Gamma$. Then we choose a rankthree translation normal subgroup $T$ in $\mathcal{G}$. The space $E^{3} / T$ is our $T^{3}$. The finite group $G=\mathcal{G} / T$ acts on $T^{3}$ preserving the graph $\Theta=\Gamma / T$. Finally, we choose an equivariant regular neighbourhood $N(\Theta)$ of $\Theta$, and $\partial N(\Theta)$ is our surface $\Sigma_{g}$. After the discussion of $\S 5$ we will see that for each example the complement of $N(\Theta)$ is also a handlebody, hence the surface is unknotted. 
Definition 3.1. Let $T_{1}=\{(a, b, c) \mid a, b, c \in \mathbb{Z}\}$ be the group of integer translations. Let $t_{x}=(1,0,0), t_{y}=(0,1,0), t_{z}=(0,0,1)$. An element $t=(a, b, c) \in T_{1}$ acts on $E^{3}$ as following:

$$
t:(x, y, z) \mapsto(x+a, y+b, z+c)
$$

For $n \in \mathbb{Z}_{+}$, we define three classes of subgroups $T_{m}$ of $T_{1}$ for those integers $m$ that can be presented (uniquely) in the form $n^{3}, 2 n^{3}$ and $4 n^{3}$ as follows:

$$
\begin{aligned}
T_{n^{3}} & =\left\langle n t_{x}, n t_{y}, n t_{z}\right\rangle \\
T_{2 n^{3}} & =\left\langle n t_{y}+n t_{z}, n t_{z}+n t_{x}, n t_{x}+n t_{y}\right\rangle, \\
T_{4 n^{3}} & =\left\langle-n t_{x}+n t_{y}+n t_{z}, n t_{x}-n t_{y}+n t_{z}, n t_{x}+n t_{y}-n t_{z}\right\rangle .
\end{aligned}
$$

The subscript $m$ of $T_{m}$ is equal to the volume $\operatorname{Vol}\left(E^{3} / T_{m}\right)$, for the above cases with $m=n^{3}, 2 n^{3}, 4 n^{3}$. We use the subscript $m$ for convenience, and note that the co-volume of a sublattice is not sufficient to uniquely identify a particular sublattice in general.

Clearly $T_{2 n^{3}}$ and $T_{4 n^{3}}$ are subgroups of $T_{n^{3}}$. One can easily verify that $T_{(2 n)^{3}} \subset T_{2 n^{3}}$, since $2 n t_{x}, 2 n t_{y}, 2 n t_{z}$ are linear combinations of $n t_{y}+n t_{z}, n t_{z}+$ $n t_{x}, n t_{x}+n t_{y}$, hence $T_{32 n^{3}}=T_{4(2 n)^{3}} \subset T_{(2 n)^{3}}=T_{8 n^{3}} \subset T_{2 n^{3}}$. Similarly one can verify that $T_{(2 n)^{3}} \subset T_{4 n^{3}}$, hence $T_{16 n^{3}}=T_{2(2 n)^{3}} \subset T_{(2 n)^{3}}=T_{8 n^{3}} \subset T_{4 n^{3}}$.

Definition 3.2. We define five isometries of $E^{3}$ as follows:

$$
\begin{aligned}
r_{y} & :(x, y, z) \\
r_{z} & :(x, y, z) \mapsto(-x, y,-z) \\
r_{x y} & :(x, y, z) \mapsto(y, x,-z) \\
r_{x y z} & :(x, y, z) \mapsto(z, x, y) \\
t_{1 / 2} & :(x, y, z) \mapsto(x+1 / 2, y+1 / 2, z+1 / 2)
\end{aligned}
$$

The isometries $r_{y}, r_{z}$ and $r_{x y}$ are 2 -fold rotations (i.e., rotation by an angle of $\pi$ ) about the $y$-axis, $z$-axis and the line $x=y, z=0$ respectively. The isometry $r_{x y z}$ is a positive 3 -fold rotation about the cube body diagonal, $x=y=z$ (i.e., right-hand rule rotation by $2 \pi / 3$ about the direction $[1,1,1]$.)

The notations of space groups used below come from the International Tables for Crystallography [Ha].

Example 3.3. Let $\Gamma_{\text {min }}^{1}$ be the one-skeleton of the unit cube $[0,1]^{3}$ in $E^{3}$ as in Figure 2. Let $\Gamma^{1}=\bigcup_{t \in T_{1}} t\left(\Gamma_{\text {min }}^{1}\right)$. Then $\Gamma^{1}$, the one-skeleton of the tessellation of $E^{3}$ by unit cubes, is a triply periodic graph called the simple or primitive cubic lattice (pcu in [RCSR]).

Let $H^{1}=\left\langle r_{y}, r_{z}, r_{x y}, r_{x y z}\right\rangle$, which is the well-known orientation-preserving isometric group of $[-1,1]^{3}$ of order 24 (with Schönflies symbol $O$ ). Let $\mathcal{G}^{1}=\left\langle T_{1}, H^{1}\right\rangle$, this is the space group $[P 432]$. Then $\mathcal{G}^{1}$ preserves $\Gamma^{1}$. Now in $T^{3} \cong E^{3} / T_{1}$ we have a graph $\Theta_{1}^{1}=\Gamma^{1} / T_{1}$. It has one vertex and three edges, hence its Euler characteristic $\chi\left(\Theta_{1}^{1}\right)=-2$ and its genus $g=1-\chi\left(\Theta_{1}^{1}\right)=3$. Clearly $G_{1}^{1}=\mathcal{G}^{1} / T_{1} \cong H^{1}$ acts on $T^{3} \cong E^{3} / T_{1}$ preserving $\Theta_{1}^{1}$, and $G_{1}^{1}$ 


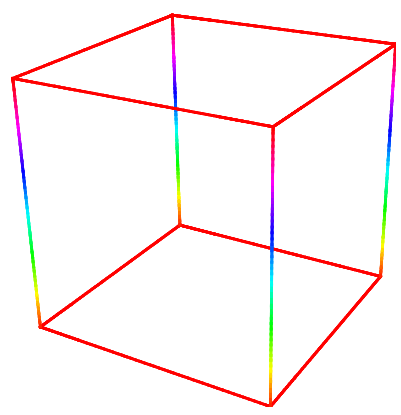

Figure 2. One-skeleton of $[0,1]^{3}$

has order 24. Hence when we choose an equivariant regular neighbourhood $N\left(\Theta_{1}^{1}\right)$ of $\Theta_{1}^{1}$, we get an extendable action of order 24 on $\Sigma_{3} \cong \partial N\left(\Theta_{1}^{1}\right)$.

Similarly in $T^{3} \cong E^{3} / T_{n^{3}}$ we have a graph $\Theta_{n^{3}}^{1}=\Gamma^{1} / T_{n^{3}}$. Since $T_{n^{3}}$ is a normal subgroup of $\mathcal{G}^{1}, G_{n^{3}}^{1}=\mathcal{G}^{1} / T_{n^{3}}$ acts on $T^{3} \cong E^{3} / T_{n^{3}}$ preserving $\Theta_{n^{3}}^{1}$.

$$
\begin{gathered}
\chi\left(\Theta_{n^{3}}^{1}\right)=\chi\left(\Theta_{1}^{1}\right) \cdot \operatorname{Vol}\left(E^{3} / T_{n^{3}}\right)=-2 n^{3} \\
\left|G_{n^{3}}^{1}\right|=\left|G_{1}^{1}\right| \cdot \operatorname{Vol}\left(E^{3} / T_{n^{3}}\right)=24 n^{3}
\end{gathered}
$$

Hence the genus of $\Theta_{n^{3}}^{1}$ is $2 n^{3}+1$. Then we can get an extendable action of order $24 n^{3}$ on $\Sigma_{2 n^{3}+1} \cong \partial N\left(\Theta_{n^{3}}^{1}\right)$.

Notice that when $m=2 n^{3}, 4 n^{3}, n \in \mathbb{Z}_{+}, T_{m}$ is also a normal subgroup of $\mathcal{G}^{1}$. Similar to the above construction, we can get an extendable action of order $24 m$ on $\Sigma_{2 m+1} \cong \partial N\left(\Theta_{m}^{1}\right)$. Here $\Theta_{m}^{1}=\Gamma^{1} / T_{m}$ is a graph in $T^{3} \cong E^{3} / T_{m}$.

In the above example the superscript 1 in $\Gamma^{1}$ or $\Theta_{m}^{1}$ is equal to the volume of the 'minimal 3-torus' $E^{3} / T_{1}$. It is also equal to the number of vertices of the graph $\Theta_{1}^{1}$. In the following examples we use similar notations.

Example 3.4. Let $\Gamma_{\min }^{2}$ be the graph in the unit cube $[0,1]^{3}$ in $E^{3}$ as in Figure 3. It consists of four edges from $(1 / 2,1 / 2,1 / 2)$ to $(0,0,0),(0,1,1)$, $(1,0,1),(1,1,0)$. Let $\Gamma^{2}=\bigcup_{t \in T_{2}} t\left(\Gamma_{\min }^{2}\right)$. One can check that it is connected and is known to scientists as the bonding structure of diamond (dia in [RCSR]). Figure 3 shows a fundamental region of $T_{2}$.

Let $H^{2}=\left\langle r_{y}, r_{z}, r_{x y z}\right\rangle$. It is the orientation-preserving isometric group of the regular tetrahedron formed by the convex hull of $(1,1,1),(1,-1,-1)$, $(-1,1,-1)$, and $(-1,-1,1)$ and has Schönflies symbol $T$. Let $\mathcal{G}^{2}=\left\langle T_{2}, H^{2}, t_{1 / 2} r_{x y}\right\rangle$, this is the space group $\left[F 4_{1} 32\right]$. Then $\mathcal{G}^{2}$ preserves $\Gamma^{2}$.

Now in $T^{3} \cong E^{3} / T_{2}$ we have a graph $\Theta_{2}^{2}=\Gamma^{2} / T_{2}$. It has two vertices and four edges, hence its Euler characteristic $\chi\left(\Theta_{2}^{2}\right)=-2$ and its genus is 3. $T_{2}$ is a normal subgroup of $\mathcal{G}^{2}$ and $G_{2}^{2}=\mathcal{G}^{2} / T_{2}$ has order 24 . It acts on $T^{3} \cong E^{3} / T_{2}$ preserving $\Theta_{2}^{2}$. Hence when we choose an equivariant regular 


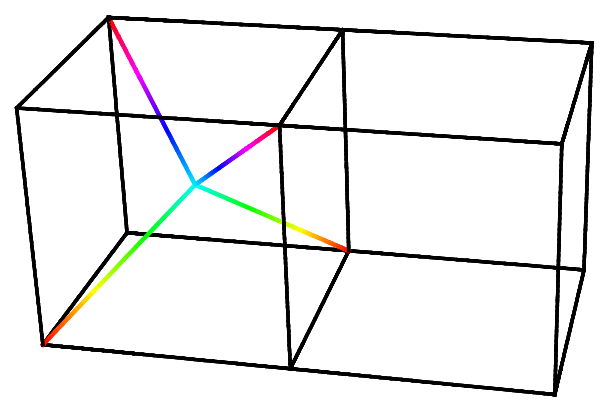

Figure $3 . \Gamma_{\min }^{2}$ in $[0,2] \times[0,1] \times[0,1]$. This is a fundamental region for $T_{2}$, but recall that the translations for $T_{2}$ are $y+z$, $z+x, x+y$, and these do not glue up directly opposite faces of the rectangular prism illustrated.

neighbourhood $N\left(\Theta_{2}^{2}\right)$ of $\Theta_{2}^{2}$, we get an extendable action of order 24 on $\Sigma_{3} \cong \partial N\left(\Theta_{2}^{2}\right)$.

Similarly when $m=n^{3}, 4 n^{3}, 16 n^{3}, n \in \mathbb{Z}_{+}$, in $T^{3} \cong E^{3} / T_{2 m}$ we have a graph $\Theta_{2 m}^{2}=\Gamma^{2} / T_{2 m}$. One can check that $T_{2 m}$ is a normal subgroup of $\mathcal{G}^{2}$, and the quotient $G_{2 m}^{2}=\mathcal{G}^{2} / T_{2 m}$ acts on $T^{3} \cong E^{3} / T_{2 m}$ preserving $\Theta_{2 m}^{2}$.

$$
\begin{gathered}
\chi\left(\Theta_{2 m}^{2}\right)=\chi\left(\Theta_{2}^{2}\right) \cdot \operatorname{Vol}\left(E^{3} / T_{2 m}\right) / \operatorname{Vol}\left(E^{3} / T_{2}\right)=-2 m \\
\left|G_{2 m}^{2}\right|=\left|G_{2}^{2}\right| \cdot \operatorname{Vol}\left(E^{3} / T_{2 m}\right) / \operatorname{Vol}\left(E^{3} / T_{2}\right)=24 m
\end{gathered}
$$

Hence the genus of $\Theta_{2 m}^{2}$ is $2 m+1$. Then we can get an extendable action of order $24 m$ on $\Sigma_{2 m+1} \cong \partial N\left(\Theta_{2 m}^{2}\right)$.

Example 3.5. Let $\gamma$ be the graph in the unit cube $[0,1]^{3}$ in $E^{3}$ as in Figure 4 . It has three edges from $(1 / 4,1 / 4,1 / 4)$ to $(0,1 / 2,1 / 4),(1 / 4,0,1 / 2)$ and $(1 / 2,1 / 4,0)$. Let $\Gamma_{\text {min }}^{4}=\gamma \cup t_{y}^{2} t_{z} r_{y} r_{z}(\gamma) \cup t_{x}^{2} t_{y} t_{z} r_{y}(\gamma) \cup t_{x}^{2} t_{y} r_{z}(\gamma)$. Then let $\Gamma^{4}=\bigcup_{t \in T_{4}} t\left(\Gamma_{\text {min }}^{4}\right)$. One can check that it is connected, and is the oft-rediscovered chiral vertex-transitive net of degree-3 known by many names $[\mathrm{HOP}]$, including srs in [RCSR]. Figure 4 shows a fundamental region of $T_{4}$.

Let $\mathcal{G}^{4}=\left\langle T_{4}, t_{x} t_{z} r_{z}, t_{y} t_{z} r_{y}, r_{x y z}, t_{x} t_{1 / 2} r_{x y}\right\rangle$, it is the space group [I4 32$]$. Then $\mathcal{G}^{4}$ preserves $\Gamma^{4}$.

Now in $T^{3} \cong E^{3} / T_{4}$ we have a graph $\Theta_{4}^{4}=\Gamma^{4} / T_{4}$. It has four vertices and six edges, hence its Euler characteristic $\chi\left(\Theta_{4}^{4}\right)=-2$ and its genus is 3. $T_{4}$ is a normal subgroup of $\mathcal{G}^{4}$ and $G_{4}^{4}=\mathcal{G}^{4} / T_{4}$ has order 24. It acts on $T^{3} \cong E^{3} / T_{4}$ preserving $\Theta_{4}^{4}$. Hence when we choose an equivariant regular neighbourhood $N\left(\Theta_{4}^{4}\right)$ of $\Theta_{4}^{4}$, we get an extendable action of order 24 on $\Sigma_{3} \cong \partial N\left(\Theta_{4}^{4}\right)$.

Similarly when $m=n^{3}, 2 n^{3}, 4 n^{3}, n \in \mathbb{Z}_{+}$, in $T^{3} \cong E^{3} / T_{4 m}$ we have a graph $\Theta_{4 m}^{4}=\Gamma^{4} / T_{4 m}$. One can check that $T_{4 m}$ is a normal subgroup of $\mathcal{G}^{4}$, 


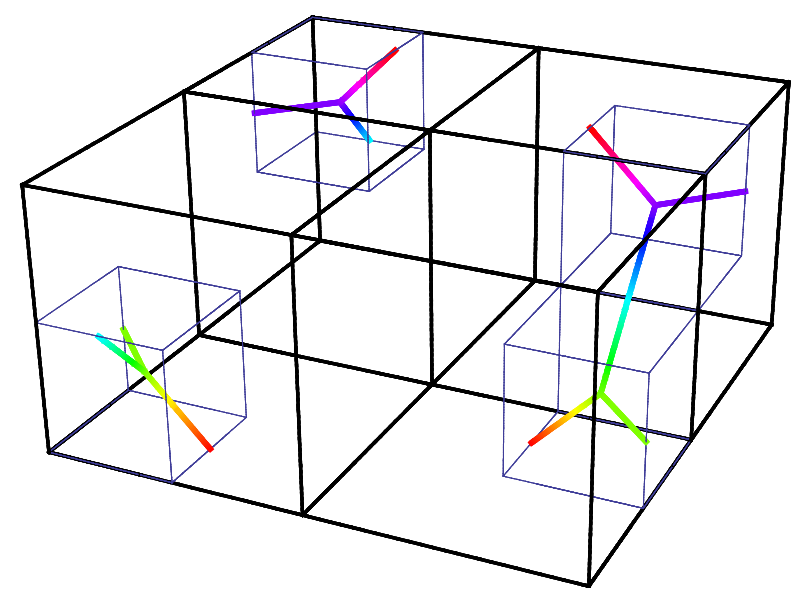

Figure $4 . \Gamma_{\min }^{4}$ in $[0,2] \times[0,2] \times[0,1]$. Similar to the previous example, this is a fundamental region for $T_{4}$ although the $T_{4}$ translations do not identify directly opposite faces of the rectangular prism illustrated.

and the quotient $G_{4 m}^{4}=\mathcal{G}^{4} / T_{4 m}$ acts on $T^{3} \cong E^{3} / T_{4 m}$ preserving $\Theta_{4 m}^{4}$.

$$
\begin{gathered}
\chi\left(\Theta_{4 m}^{4}\right)=\chi\left(\Theta_{4}^{4}\right) \cdot \operatorname{Vol}\left(E^{3} / T_{4 m}\right) / \operatorname{Vol}\left(E^{3} / T_{4}\right)=-2 m \\
\left|G_{4 m}^{4}\right|=\left|G_{4}^{4}\right| \cdot \operatorname{Vol}\left(E^{3} / T_{4 m}\right) / \operatorname{Vol}\left(E^{3} / T_{4}\right)=24 m
\end{gathered}
$$

Hence the genus of $\Theta_{4 m}^{4}$ is $2 m+1$. Then we can get an extendable action of order $24 m$ on $\Sigma_{2 m+1} \cong \partial N\left(\Theta_{4 m}^{4}\right)$.

\section{KNotTed EXAmples of the MAXIMALly SymmetriC SURFACES}

In the section we give six further classes of examples realizing the upper bound of $E_{+}^{+}\left(\Sigma_{g}\right)=12(g-1)$. In each case below the embedding is knotted. The surface $e\left(\Sigma_{g}\right)$ does bound a handlebody in $T^{3}$ on one side, but the complement of the handlebody is not a handlebody, and this will be clear after the discussion of $\S 5$.

Constructions of the extendable actions and surfaces is similar to $\S 4$, but here the graph $\Gamma$ in $E^{3}$ can be disconnected. There is a space group $\mathcal{G}$ preserving $\Gamma$ and a rank three translation normal subgroup $T$ in $\mathcal{G}$ so that the graph $\Theta=\Gamma / T$ is connected. The finite group $G=\mathcal{G} / T$ acts on $T^{3} \cong E^{3} / T$ preserving $\Theta$. Then we choose an equivariant regular neighbourhood $N(\Theta)$ of $\Theta$, and $\partial N(\Theta)$ is our surface $\Sigma_{g}$.

Example 4.1. Let $\Gamma^{1,2}=\Gamma^{1} \cup t_{1 / 2}\left(\Gamma^{1}\right)$. The graph $t_{1 / 2}\left(\Gamma^{1}\right)$ can be thought of as the dual of $\Gamma^{1}$ in $E^{3}$. $\Gamma^{1,2}$ has two connected components and is named pcu-c in $[\mathrm{RCSR}]$. In the unit cube $[0,1]^{3}$ it is as in Figure 5. 


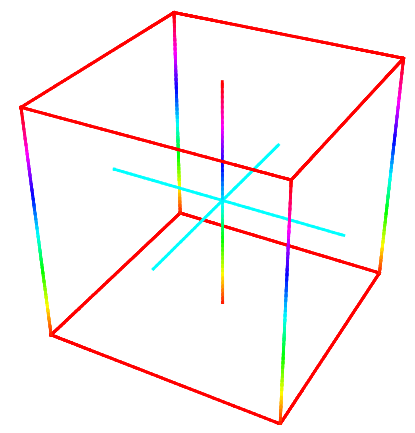

Figure 5. Part of $\Gamma^{1,2}$ in $[0,1]^{3}$

Let $\mathcal{G}^{1,2}=\left\langle\mathcal{G}^{1}, t_{1 / 2}\right\rangle$, it is the space group [I432], and let $T_{1 / 2}=\left\langle T_{1}, t_{1 / 2}\right\rangle=$ $\left\{(a / 2, b / 2, c / 2) \mid(a, b, c) \in T_{4}\right\}$. Then $\mathcal{G}^{1,2}$ preserves $\Gamma^{1,2}$ and $T_{1 / 2}$ is a normal subgroup of $\mathcal{G}^{1,2}$. Since $t_{1 / 2}$ changes the two components of $\Gamma^{1,2}$, the graph $\Theta_{1 / 2}^{1,2}=\Gamma^{1,2} / T_{1 / 2}$ in $T^{3} \cong E^{3} / T_{1 / 2}$ is connected. Its Euler characteristic $\chi\left(\Theta_{1 / 2}^{1,2}\right)=-2$ and its genus is $3 . G_{1 / 2}^{1,2}=\mathcal{G}^{1,2} / T_{1 / 2}$ has order 24 . It acts on $T^{3} \cong E^{3} / T_{1 / 2}$ preserving $\Theta_{1 / 2}^{1,2}$. Hence when we choose an equivariant regular neighbourhood $N\left(\Theta_{1 / 2}^{1,2}\right)$ of $\Theta_{1 / 2}^{1,2}$, we get an extendable action of order 24 on $\Sigma_{3} \cong \partial N\left(\Theta_{1 / 2}^{1,2}\right)$.

Similarly let $T_{n^{3} / 2}=\left\{(a / 2, b / 2, c / 2) \mid(a, b, c) \in T_{4 n^{3}}\right\}, n \in \mathbb{Z}_{+}, 2 \nmid n$. Then the graph $\Theta_{n^{3} / 2}^{1,2}=\Gamma^{1,2} / T_{n^{3} / 2}$ in $T^{3} \cong E^{3} / T_{n^{3} / 2}$ is connected. Since $T_{n^{3} / 2}$ is a normal subgroup of $\mathcal{G}^{1,2}, G_{n^{3} / 2}^{1,2}=\mathcal{G}^{1,2} / T_{n^{3} / 2}$ acts on $T^{3} \cong$ $E^{3} / T_{n^{3} / 2}$ preserving $\Theta_{n^{3} / 2}^{1,2}$.

$$
\begin{gathered}
\chi\left(\Theta_{n^{3} / 2}^{1,2}\right)=\chi\left(\Theta_{1 / 2}^{1,2}\right) \cdot \operatorname{Vol}\left(E^{3} / T_{n^{3} / 2}\right) / \operatorname{Vol}\left(E^{3} / T_{1 / 2}\right)=-2 n^{3} \\
\left|G_{n^{3} / 2}^{1,2}\right|=\left|G_{1 / 2}^{1,2}\right| \cdot \operatorname{Vol}\left(E^{3} / T_{n^{3} / 2}\right) / \operatorname{Vol}\left(E^{3} / T_{1 / 2}\right)=24 n^{3}
\end{gathered}
$$

Hence the genus of $\Theta_{n^{3} / 2}^{1,2}$ is $2 n^{3}+1$. Then we can get an extendable action of order $24 n^{3}$ on $\Sigma_{2 n^{3}+1} \cong \partial N\left(\Theta_{n^{3} / 2}^{1,2}\right)$, here $n \in \mathbb{Z}_{+}, 2 \nmid n$.

Example 4.2. Let $\Gamma^{2,2}=\Gamma^{2} \cup t_{x}\left(\Gamma^{2}\right)$. The graph $t_{x}\left(\Gamma^{2}\right)$ can be thought as the dual of $\Gamma^{2}$ in $E^{3}$. $\Gamma^{2,2}$ has two connected components and is called dia-c in [RCSR]. In the fundamental region of $T_{2}$ it is as in Figure 6.

Let $\mathcal{G}^{2,2}=\left\langle\mathcal{G}^{2}, t_{x}\right\rangle$, it is the space group $\left[P 4_{2} 32\right]$. Then $\mathcal{G}^{2,2}$ preserves $\Gamma^{2,2}$ and contains $T_{1}$ as a normal subgroup. Since $t_{x}$ changes the two components of $\Gamma^{2,2}$, the graph $\Theta_{1}^{2,2}=\Gamma^{2,2} / T_{1}$ in $T^{3} \cong E^{3} / T_{1}$ is connected. Its Euler characteristic $\chi\left(\Theta_{1}^{2,2}\right)=-2$, and its genus is $3 . G_{1}^{2,2}=\mathcal{G}^{2,2} / T_{1}$ has order 24. It acts on $T^{3} \cong E^{3} / T_{1}$ preserving $\Theta_{1}^{2,2}$. Hence when we choose an 


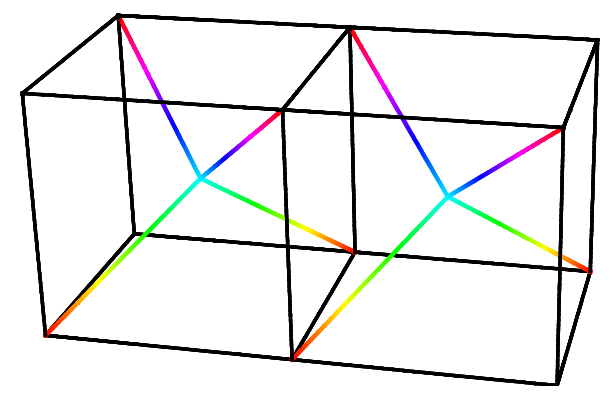

Figure 6. Part of $\Gamma^{2,2}$ in $[0,2] \times[0,1] \times[0,1]$

equivariant regular neighbourhood $N\left(\Theta_{1}^{2,2}\right)$ of $\Theta_{1}^{2,2}$, we get an extendable action of order 24 on $\Sigma_{3} \cong \partial N\left(\Theta_{1}^{2,2}\right)$.

Similarly if $m=n^{3}, 4 n^{3}, n \in \mathbb{Z}_{+}, 2 \nmid n$, the graph $\Theta_{m}^{2,2}=\Gamma^{2,2} / T_{m}$ in $T^{3} \cong E^{3} / T_{m}$ is connected. One can check that $T_{m}$ is a normal subgroup of $\mathcal{G}^{2,2}, G_{m}^{2,2}=\mathcal{G}^{2,2} / T_{m}$ acts on $T^{3} \cong E^{3} / T_{m}$ preserving $\Theta_{m}^{2,2}$.

$$
\begin{gathered}
\chi\left(\Theta_{m}^{2,2}\right)=\chi\left(\Theta_{1}^{2,2}\right) \cdot \operatorname{Vol}\left(E^{3} / T_{m}\right)=-2 m \\
\left|G_{m}^{2,2}\right|=\left|G_{1}^{2,2}\right| \cdot \operatorname{Vol}\left(E^{3} / T_{m}\right)=24 m
\end{gathered}
$$

Hence the genus of $\Theta_{m}^{2,2}$ is $2 m+1$. Then we can get an extendable action of order $24 m$ on $\Sigma_{2 m+1} \cong \partial N\left(\Theta_{m}^{2,2}\right)$, here $m=n^{3}, 4 n^{3}, n \in \mathbb{Z}_{+}, 2 \nmid n$.

Example 4.3. Let $\Gamma^{4,4}=\Gamma^{4} \cup t_{x}\left(\Gamma^{4}\right) \cup t_{y}\left(\Gamma^{4}\right) \cup t_{z}\left(\Gamma^{4}\right) . \quad \Gamma^{4,4}$ has four connected components. In the unit cube $[0,1]^{3}$ in $E^{3}$ it is as in Figure 7.

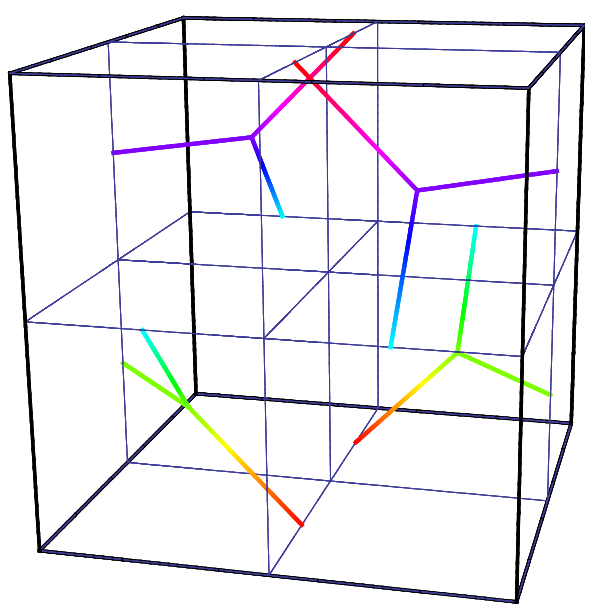

Figure 7. Part of $\Gamma^{4,4}$ in $[0,1]^{3}$ 
One can check that $\mathcal{G}^{2,2}$ defined in Example 4.2 (space group $\left[P 4_{2} 32\right]$ ) preserves $\Gamma^{4,4}$, and the graph $\Theta_{1}^{4,4}=\Gamma^{4,4} / T_{1}$ in $T^{3} \cong E^{3} / T_{1}$ is connected. Its Euler characteristic $\chi\left(\Theta_{1}^{4,4}\right)=-2$, and its genus is $3 . G_{1}^{2,2}=\mathcal{G}^{2,2} / T_{1}$ has order 24 . It acts on $T^{3} \cong E^{3} / T_{1}$ preserving $\Theta_{1}^{4,4}$. Hence when we choose an equivariant regular neighbourhood $N\left(\Theta_{1}^{4,4}\right)$ of $\Theta_{1}^{4,4}$, we get an extendable action of order 24 on $\Sigma_{3} \cong \partial N\left(\Theta_{1}^{4,4}\right)$.

Similarly if $m=n^{3}, 2 n^{3}, n \in \mathbb{Z}_{+}, 2 \nmid n$, the graph $\Theta_{m}^{4,4}=\Gamma^{4,4} / T_{m}$ in $T^{3} \cong E^{3} / T_{m}$ is connected. $G_{m}^{2,2}$ acts on $T^{3} \cong E^{3} / T_{m}$ preserving $\Theta_{m}^{4,4}$.

$$
\begin{gathered}
\chi\left(\Theta_{m}^{4,4}\right)=\chi\left(\Theta_{1}^{4,4}\right) \cdot \operatorname{Vol}\left(E^{3} / T_{m}\right)=-2 m \\
\left|G_{m}^{2,2}\right|=\left|G_{1}^{2,2}\right| \cdot \operatorname{Vol}\left(E^{3} / T_{m}\right)=24 m
\end{gathered}
$$

Hence the genus of $\Theta_{m}^{4,4}$ is $2 m+1$. Then we can get an extendable action of order $24 m$ on $\Sigma_{2 m+1} \cong \partial N\left(\Theta_{m}^{2,2}\right)$, here $m=n^{3}, 2 n^{3}, n \in \mathbb{Z}_{+}, 2 \nmid n$.

Example 4.4. Let $\Gamma^{4,8}=\Gamma^{4,4} \cup t_{1 / 2}\left(\Gamma^{4,4}\right)$. It has eight connected components. In the unit cube $[0,1]^{3}$ it is as in Figure 8 .

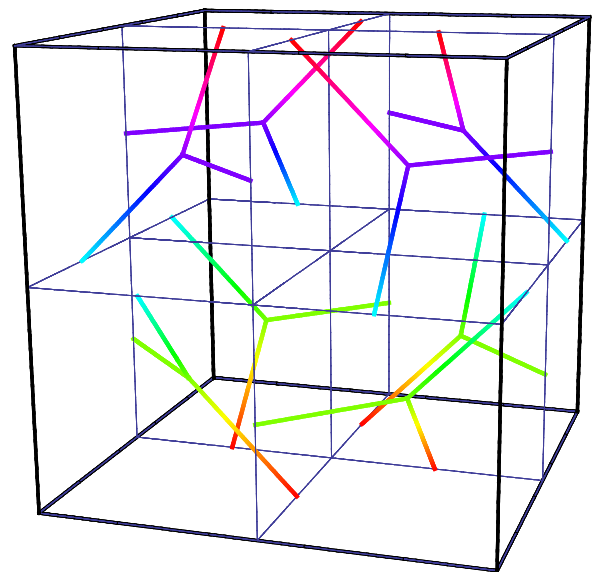

Figure 8. Part of $\Gamma^{4,8}$ in $[0,1]^{3}$

One can check that $\mathcal{G}^{1,2}$ defined in Example 4.1 (space group [I432]) preserves $\Gamma^{4,8}$, and the graph $\Theta_{1 / 2}^{4,8}=\Gamma^{4,8} / T_{1 / 2}$ in $T^{3} \cong E^{3} / T_{1 / 2}$ is connected. Its Euler characteristic is -2 and its genus is 3 . The order 24 group $G_{1 / 2}^{1,2}$ acts on $T^{3} \cong E^{3} / T_{1 / 2}$ preserving $\Theta_{1 / 2}^{4,8}$. Hence when we choose an equivariant regular neighbourhood $N\left(\Theta_{1 / 2}^{4,8}\right)$ of $\Theta_{1 / 2}^{4,8}$, we get an extendable action of order 24 on $\Sigma_{3} \cong \partial N\left(\Theta_{1 / 2}^{4,8}\right)$. 
Similarly for $n \in \mathbb{Z}_{+}, 2 \nmid n$, the graph $\Theta_{n^{3} / 2}^{4,8}=\Gamma^{4,8} / T_{n^{3} / 2}$ in $T^{3} \cong$ $E^{3} / T_{n^{3} / 2}$ is connected. $G_{n^{3} / 2}^{1,2}$ acts on $T^{3} \cong E^{3} / T_{n^{3} / 2}$ preserving $\Theta_{n^{3} / 2}^{4,8}$.

$$
\begin{gathered}
\chi\left(\Theta_{n^{3} / 2}^{4,8}\right)=\chi\left(\Theta_{1 / 2}^{4,8}\right) \cdot \operatorname{Vol}\left(E^{3} / T_{n^{3} / 2}\right) / \operatorname{Vol}\left(E^{3} / T_{1 / 2}\right)=-2 n^{3} \\
\left|G_{n^{3} / 2}^{1,2}\right|=\left|G_{1 / 2}^{1,2}\right| \cdot \operatorname{Vol}\left(E^{3} / T_{n^{3} / 2}\right) / \operatorname{Vol}\left(E^{3} / T_{1 / 2}\right)=24 n^{3}
\end{gathered}
$$

Hence the genus of $\Theta_{n^{3} / 2}^{4,8}$ is $2 n^{3}+1$. Then we can get an extendable action of order $24 n^{3}$ on $\Sigma_{2 n^{3}+1} \cong \partial N\left(\Theta_{n^{3} / 2}^{4,8}\right)$, here $n \in \mathbb{Z}_{+}, 2 \nmid n$.

Example 4.5. Let $\gamma^{\prime}$ be the graph in the unit cube $[0,1]^{3}$ in $E^{3}$ as in Figure 4. There are three edges from $(1 / 4,1 / 4,1 / 4)$ to $(0,1 / 4,1 / 2),(1 / 2,0,1 / 4)$, $(1 / 4,1 / 2,0)$, and three edges from $(0,3 / 4,1 / 2)$ to $(1 / 2,3 / 4,1),(1 / 2,0,3 / 4)$ to $(1,1 / 2,3 / 4)$ and $(3 / 4,1 / 2,0)$ to $(3 / 4,1,1 / 2)$ separately.

Let $\Gamma_{\text {min }}^{\prime 4}=\gamma^{\prime} \cup t_{y}^{2} t_{z} r_{y} r_{z}\left(\gamma^{\prime}\right) \cup t_{x}^{2} t_{y} t_{z} r_{y}\left(\gamma^{\prime}\right) \cup t_{x}^{2} t_{y} r_{z}\left(\gamma^{\prime}\right)$, and $\Gamma^{\prime 4}=\bigcup_{t \in T_{4}} t\left(\Gamma_{\text {min }}^{\prime 4}\right)$. One can check that $\Gamma^{4}$ has 27 connected components, each is similar to a mirror image of $\Gamma^{4}$. Figure 9 shows the part of $\Gamma^{\prime 4}$ in a fundamental region of $T_{4}$.

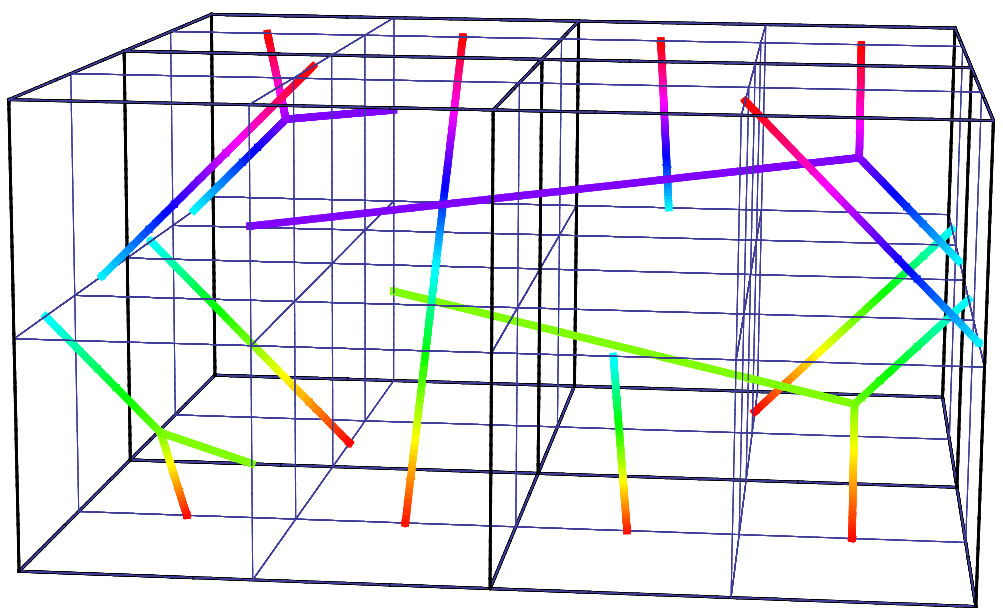

Figure 9. Part of $\Gamma^{4} \min$ in $[0,2] \times[0,2] \times[0,1]$

By construction, $\mathcal{G}^{4}$ (space group $\left[I 4_{1} 32\right]$ ) preserves $\Gamma^{4}$. And one can check that $\Theta_{4}^{\prime 4}=\Gamma^{\prime 4} / T_{4}$ in $T^{3} \cong E^{3} / T_{4}$ is connected. $\Theta_{4}^{\prime 4}$ has four vertices and six edges, hence it has Euler characteristic -2 and genus 3 . The order24 group $G_{4}^{4}$ acts on $T^{3} \cong E^{3} / T_{4}$ preserving $\Theta_{4}^{\prime 4}$. Hence when we choose an equivariant regular neighbourhood $N\left(\Theta_{4}^{\prime 4}\right)$ of $\Theta_{4}^{\prime 4}$, we get an extendable action of order 24 on $\Sigma_{3} \cong \partial N\left(\Theta_{4}^{\prime 4}\right)$.

Similarly when $m=n^{3}, 2 n^{3}, 4 n^{3}, n \in \mathbb{Z}_{+}, 3 \nmid n$, the graph $\Theta_{4 m}^{\prime 4}=\Gamma^{\prime 4} / T_{4 m}$ in $T^{3} \cong E^{3} / T_{4 m}$ is connected. The quotient $G_{4 m}^{4}=\mathcal{G}^{4} / T_{4 m}$ acts on $T^{3} \cong$ 
$E^{3} / T_{4 m}$ preserving $\Theta_{4 m}^{\prime 4}$.

$$
\begin{gathered}
\chi\left(\Theta_{4 m}^{\prime 4}\right)=\chi\left(\Theta_{4}^{\prime 4}\right) \cdot \operatorname{Vol}\left(E^{3} / T_{4 m}\right) / \operatorname{Vol}\left(E^{3} / T_{4}\right)=-2 m \\
\left|G_{4 m}^{4}\right|=\left|G_{4}^{4}\right| \cdot \operatorname{Vol}\left(E^{3} / T_{4 m}\right) / \operatorname{Vol}\left(E^{3} / T_{4}\right)=24 m
\end{gathered}
$$

Hence the genus of $\Theta_{4 m}^{\prime 4}$ is $2 m+1$. Then we can get an extendable action of order $24 m$ on $\Sigma_{2 m+1} \cong \partial N\left(\Theta_{4 m}^{\prime 4}\right)$, here $m=n^{3}, 2 n^{3}, 4 n^{3}, n \in \mathbb{Z}_{+}, 3 \nmid n$.

Definition 4.6. Let $t_{\omega}=(-1 / 2, \sqrt{3} / 2,0)$. Define

$$
\begin{aligned}
T_{n^{2}}^{\omega} & =\left\langle n t_{\omega}, n t_{x}, t_{z}\right\rangle, \\
T_{3 n^{2}}^{\omega} & =\left\langle 2 n t_{\omega}+n t_{x}, n t_{\omega}+2 n t_{x}, t_{z}\right\rangle .
\end{aligned}
$$

Define a rotation $r_{\omega}$ on $E^{3}$ as following:

$$
r_{\omega}:(x, y, z) \mapsto\left(-\frac{1}{2} x-\frac{\sqrt{3}}{2} y, \frac{\sqrt{3}}{2} x-\frac{1}{2} y, z\right)
$$

$T_{1}^{\omega}$ is the hexagonal 3D lattice and $r_{\omega}$ is a rotation by $2 \pi / 3$ right-handed with respect to the direction $[0,0,1]$.

Example 4.7. Let $P$ be a regular hexagon in the $x y$-plane. It has center $(0,0,0)$ and contains $(1 / 2, \sqrt{3} / 6,0)$ as a vertex, see Figure 10 . Let $\Gamma_{\min }^{P}$ be the boundary of $P$ in $E^{3}$. Let $\Gamma^{P}=\bigcup_{t \in T_{1}^{\omega}} t\left(\Gamma_{\text {min }}^{P}\right)$. It contains infinitely many connected components, and for each $n \in \mathbb{Z}$, the horizontal plane $\left\{(x, y, n) \mid x, y \in E^{2}\right\}$ contains exactly one of them. A local picture of $\Gamma^{P}$ is as in Figure 11.

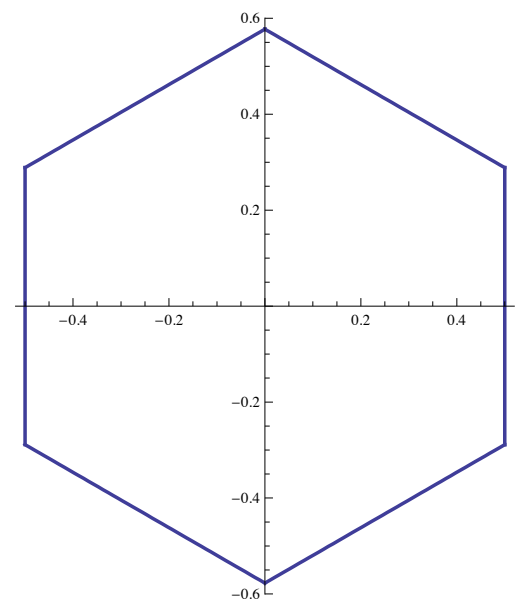

Figure 10. A regular hexagon

Let $H^{\omega}=\left\langle r_{\omega}, r_{x}, r_{y}\right\rangle$; it is the isometric group of $P$ (Schönflies symbol $\left.D_{6}\right)$. Let $\mathcal{G}^{\omega}=\left\langle T_{1}^{\omega}, H^{\omega}\right\rangle$, this is the space group [P622]. Then $\mathcal{G}^{\omega}$ preserves $\Gamma^{P}$, and $\Theta_{1}^{P}=\Gamma^{P} / T_{1}^{\omega}$ is a connected graph in $T^{3} \cong E^{3} / T_{1}^{\omega}$. It has two 


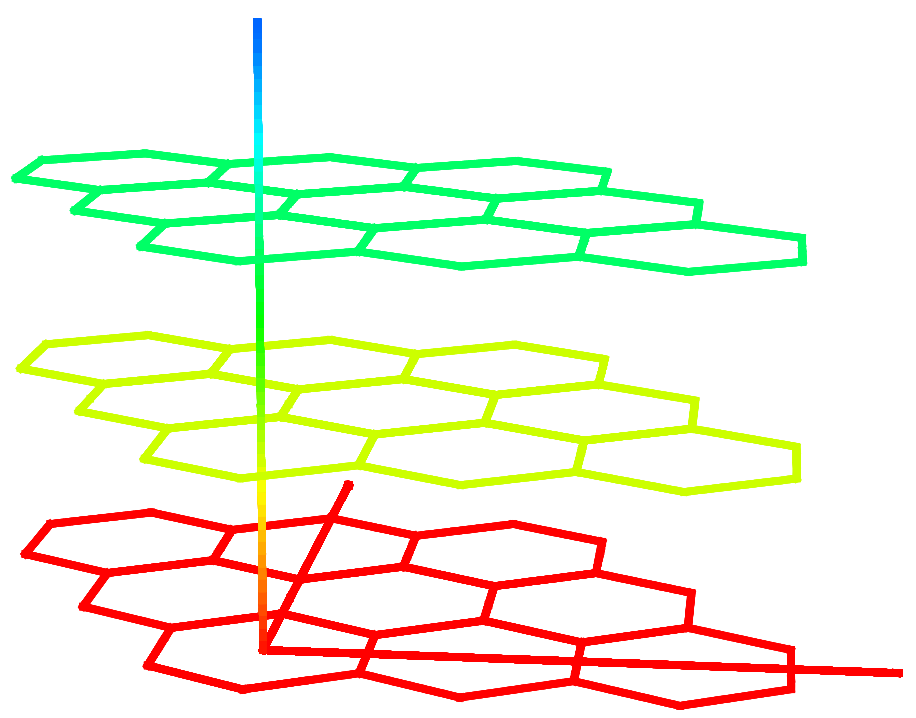

Figure 11. A local picture of $\Gamma^{P}$

vertices and three edges, hence its Euler characteristic $\chi\left(\Theta_{1}^{\omega}\right)=-1$ and its genus is 2 . Clearly $G_{1}^{\omega}=\mathcal{G}^{\omega} / T_{1}^{\omega}$ acts on $T^{3} \cong E^{3} / T_{1}^{\omega}$ preserving $\Theta_{1}^{P}$, and $G_{1}^{\omega} \cong H^{\omega}$ has order 12 . Hence when we choose an equivariant regular neighbourhood $N\left(\Theta_{1}^{P}\right)$ of $\Theta_{1}^{P}$, we get an extendable action of order 12 on $\Sigma_{2} \cong \partial N\left(\Theta_{1}^{P}\right)$.

Notice that when $m=n^{2}, 3 n^{2}, n \in \mathbb{Z}_{+}, T_{m}^{\omega}$ is a normal subgroup of $\mathcal{G}^{\omega}$. Similarly the graph $\Theta_{m}^{P}=\Gamma^{P} / T_{m}^{\omega}$ in $T^{3} \cong E^{3} / T_{m}^{\omega}$ is connected, and $G_{m}^{\omega}=\mathcal{G}^{\omega} / T_{m}^{\omega}$ acts on $T^{3} \cong E^{3} / T_{m}^{\omega}$ preserving $\Theta_{m}^{P}$.

$$
\begin{gathered}
\chi\left(\Theta_{m}^{P}\right)=\chi\left(\Theta_{1}^{P}\right) \cdot \operatorname{Vol}\left(E^{3} / T_{m}^{\omega}\right) / \operatorname{Vol}\left(E^{3} / T_{1}^{\omega}\right)=-m \\
\left|G_{m}^{\omega}\right|=\left|G_{1}^{\omega}\right| \cdot \operatorname{Vol}\left(E^{3} / T_{m}^{\omega}\right) / \operatorname{Vol}\left(E^{3} / T_{1}^{\omega}\right)=12 m
\end{gathered}
$$

Hence the genus of $\Theta_{m}^{P}$ is $m+1$. Then we can get an extendable action of order $12 m$ on $\Sigma_{m+1} \cong \partial N\left(\Theta_{m}^{P}\right)$.

\section{Minimal surfaces, space groups, proof of the main Result}

In this section we will finish the proof of Theorem 1.5. We need some results about triply periodic minimal surfaces, space groups, and a lemma given below.

There are three classical triply periodic minimal surfaces that admit high symmetries: Schwarz's P surface, Schwarz's D [Schw] and Schoen's gyroid surface illustrated in Figure $12[\mathrm{Br}]$. Denote them by $S_{P}, S_{D}$ and $S_{G}$. For each surface there is a side-preserving translational lattice such that the corresponding quotient surface has genus three. A fundamental region for the lattice in the case of $S_{P}$ is illustrated in Figure 12. A fundamental region 
for $S_{D}$ has volume two in the coordinate system of Section 3. In Figure 12 we draw $S_{D}$ in the region $[0,1] \times[0,2] \times[0,1]$ to match the domain used in Figure 3. A fundamental region for $S_{G}$ has volume four, but in Figure 12 we draw $S_{G}$ in the domain $[0,2] \times[0,2] \times[0,2]$, twice the volume of the fundamental region used in Figure 4 to assist in visualisation, and because this is the standard non-primitive cubic unit cell for the space group $\left[I 4_{1} 32\right]$.

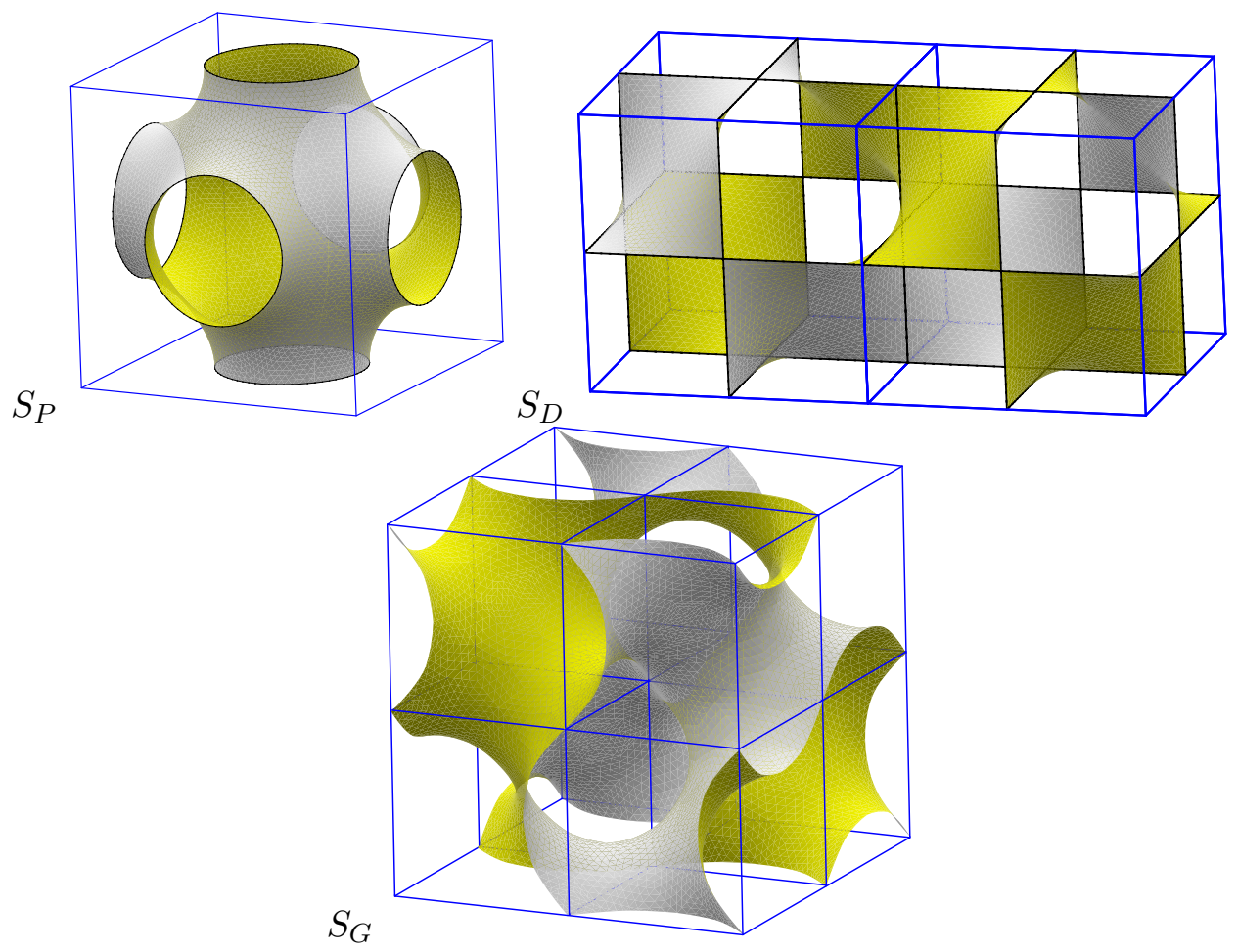

Figure 12. Translational unit cells of the minimal surfaces $S_{P}$, and $S_{D}$ match the translational unit cells of their associated graphs, $\Gamma^{1}, \Gamma^{2}$, and are displayed with the same viewing direction. The picture of $S_{G}$ above shows the cube $[0,2] \times[0,2] \times[0,2]$, twice the region of that used to depict $\Gamma^{4}$, but depicted with the same viewing direction.

The following results are known (and can be checked):

(a) Boundaries of equivariant regular neighbourhoods of $\Gamma^{1}, \Gamma^{2}$ and $\Gamma^{4}$ in Example 3.3, 3.4 and 3.5 can be realized by $S_{P}, S_{D}$ and $S_{G}$ respectively.

(b) $S_{P}, S_{D}$ and $S_{G}$ are unknotted in $T^{3}$. Actually, any genus $g>1$ minimal orientable closed surface in $T^{3}$ must be unknotted,[Me].

The notations of space groups below come from [Ha].

(c) $\mathcal{G}^{1}, \mathcal{G}^{2}, \mathcal{G}^{4}, \mathcal{G}^{1,2}$ and $\mathcal{G}^{2,2}$ in Example 3.3, 3.4, 3.5, 4.1 and 4.2 are the space groups $[P 432],\left[F 4_{1} 32\right],\left[I 4_{1} 32\right],[I 432]$ and $\left[P 4_{2} 32\right]$ respectively. 
(d) If we allow isometries that do not preserve orientations of the surface or of space then we find $S_{P}, S_{D}, S_{G}$ are preserved by space groups $[\operatorname{Im} \overline{3} m]$, $[\operatorname{Pn} \overline{3} m],[I a \overline{3} d]$ respectively.

(e) We have the following index two subgroup sequences:

- $[P 432] \subset[I 432] \subset[\operatorname{Im} \overline{3} m]$

- $[P 432] \subset[P m \overline{3} m] \subset[\operatorname{Im} \overline{3} m]$

- $\left[F 4_{1} 32\right] \subset\left[P 4_{2} 32\right] \subset[P n \overline{3} m]$

- $\left[I 4_{1} 32\right] \subset[I a \overline{3} d]$

Lemma 5.1. Let $T$ be a lattice in $E^{3}, p: E^{3} \rightarrow T^{3}=E^{3} / T$ be the covering map. $\Sigma_{g}$ is an embedded surface in $T^{3}$. If $p^{-1}\left(\Sigma_{g}\right)$ is not connected, then $\Sigma_{g}$ is knotted.

Proof. Note first, from the definition of unknotted embedding $\Sigma_{g} \subset T^{3}$, the induced map on the fundamental groups must be surjective. Let $F$ be a connected component of $p^{-1}\left(\Sigma_{g}\right)$. Let $S t(F)$ be its stable subgroup in $T$, i.e., the lattice translations that preserve $F$. If $p^{-1}\left(\Sigma_{g}\right)$ has more than one component then $S t(F) \neq T$, hence $\pi_{1}\left(\Sigma_{g}\right) \rightarrow \pi_{1}\left(T^{3}\right)$ is not surjective. Hence $\Sigma_{g}$ is knotted in $T^{3}$.

Proof of Theorem 1.5. By the above results (a) and (b), the surfaces in $T^{3}$ described in $\S 3$ are unknotted. Since $\Gamma^{1,2}, \Gamma^{2,2}, \Gamma^{4,4}, \Gamma^{4,8}, \Gamma^{4}$ and $\Gamma^{P}$ are disconnected, by Lemma 5.1, the surfaces in $T^{3}$ described in $\S 4$ are knotted. Then by combining the Examples in $\S 3$ and $\S 4$, we finish the proof of Theorem 1.5 (1).

Now we are going to prove Theorem 1.5 (2) and (3) based on the examples in $\S 3$ and $\S 4$, and the results (c), (d) and (e) above.

For $(2)$, recall $\mathcal{G}^{1}=[P 432]$. Example 3.3 told us that when $m=n^{3}, 2 n^{3}, 4 n^{3}$ we have the action $\mathcal{G}^{1} / T_{m}$ on $\Gamma^{1} / T_{m}$, or equivalently on $S_{P} / T_{m}$ by (a), of order $24 m$. By (d) $[\operatorname{Im} \overline{3} m]$ preserves $S_{P}$. By (e), we have an order $96 m$ extendable $[\operatorname{Im} \overline{3} m] / T_{m}$-action on $S_{P} / T_{m} \cong \Sigma_{2 m+1}$ realizing the upper bound of $E\left(\Sigma_{g}\right)$, where $g=2 m+1$.

The group $[\operatorname{Im} \overline{3} m] / T_{m}$ contains two elements $h_{1}$ and $h_{2}$ satisfying: $h_{1}$ preserves the orientation of $S_{P} / T_{m}$ and reverses the orientation of $E^{3} / T_{m}$ (e.g. reflection in a plane transverse to $S_{P}$ ) and $h_{2}$ reverses the orientation of $S_{P} / T_{m}$ and preserves the orientation of $E^{3} / T_{m}$ (e.g. rotation by $\pi$ about an axis that lies in $S_{P}$ ). Example choices for these elements are

$$
\begin{aligned}
& h_{1}:(x, y, z) \mapsto(1-x, y, z) \\
& h_{2}:(x, y, z) \mapsto(-x+1 / 2,-z+1 / 2,-y+1 / 2)
\end{aligned}
$$

Then choosing the index two subgroups of $[\operatorname{Im} \overline{3} m] / T_{m},\left\langle[P 432], h_{1}\right\rangle / T_{m}=$ $[P m \overline{3} m] / T_{m}$ and $\left\langle[P 432], h_{2}\right\rangle / T_{m}=[I 432] / T_{m}$, we get an extendable action on $\Sigma_{2 m+1}$, realizing the upper bound of $E_{+}\left(\Sigma_{g}\right)$ and $E^{+}\left(\Sigma_{g}\right)$ respectively. Now (2) is proved.

For $(3)$, recall $\mathcal{G}^{2}=\left[F 4_{1} 32\right]$. Example 3.4 told us that when $m=$ $n^{3}, 4 n^{3}, 16 n^{3}$ we have the action $\mathcal{G}^{2} / T_{2 m}$ on $\Gamma^{2} / T_{2 m}$, or equivalently on 
$S_{D} / T_{2 m}$ by (a), of order $24 m$. Similar to the case for $S_{P}$ above, we also have an order $96 m$ extendable $[P n \overline{3} m] / T_{2 m}$-action on $S_{D} / T_{2 m} \cong \Sigma_{2 m+1}$ realizing the upper bound of $E\left(\Sigma_{g}\right)$, where $g=2 m+1$.

Furthermore, if $m=n^{3}, 4 n^{3}, n \in \mathbb{Z}_{+}, n$ odd, $[P n \overline{3} m] / T_{2 m}$ contains a translation $h$ reversing an orientation of $S_{D} / T_{2 m}$ and preserving an orientation of $E^{3} / T_{2 m}$ (see Example 4.2 for details, or see $S_{D}$ in Figure 12 where $h$ is the translation $\left.t_{x}\right)$. Modulo this translation we can get an order $48 \mathrm{~m}$ extendable $[P n \overline{3} m] / T_{m}$-action on $S_{D} / T_{m} \cong \Pi_{2 m+2}$, realizing the upper bound $24(g-2)$ of $E\left(\Pi_{g}\right)$, where $g=2 m+2$. Then choosing the index two subgroup $\left[P 4_{2} 32\right] \subset[P n \overline{3} m]$, we can get an extendable action on $\Pi_{2 m+2}$, realizing the upper bound of $E^{+}\left(\Pi_{g}\right)$. Now (3) is proved.

Remark 5.2. In the above discussion for (3), the regular neighbourhood $N\left(S_{D} / T_{m}\right)$ of $S_{D} / T_{m}$ is homeomorphic to a twisted [-1,1]-bundle over $S_{D} / T_{m}$. Its complement in $E^{3} / T_{m}$ is essentially the regular neighbourhood $N\left(\Theta_{m}^{2,2}\right)$ of $\Theta_{m}^{2,2}$ in Example 4.2. Similarly for $n \in \mathbb{Z}_{+}, n$ odd, and $m=n^{3} / 2$, we can get an order $48 m$ extendable $[\operatorname{Im} \overline{3} m] / T_{n^{3} / 2^{-a c t i o n} \text { on }}$ $S_{P} / T_{n^{3} / 2} \cong \Pi_{2 n^{3}+2}$, realizing the upper bound of $E\left(\Pi_{g}\right)$. Choosing the index two subgroup [I432], we can get an extendable action on $\Pi_{2 n^{3}+2}$, realizing the upper bound of $E^{+}\left(\Pi_{g}\right)$. The regular neighbourhood $N\left(S_{P} / T_{n^{3} / 2}\right)$ is homeomorphic to a twisted [-1,1]-bundle over $S_{P} / T_{n^{3} / 2}$. Its complement in $E^{3} / T_{n^{3} / 2}$ is essentially the regular neighbourhood $N\left(\Theta_{n^{3} / 2}^{1,2}\right)$ in Example 4.1. There is no similar construction for a regular neighbourhood of the Gyroid minimal surface because there is no translation that reverses the orientation of $S_{G} / T_{4}$; the handlebodies on each side of $S_{G}$ are mirror images of one another.

\section{REFERENCES}

[Br] K. Brakke, Minimal surface page, http://www.susqu.edu/brakke/evolver/examples/periodic/periodic.html

[FH] C. Frohman, J. Hass, Unstable minimal surfaces and Heegaard splittings. Invent. Math. 95 (1989), no. 3, 529-540.

[Ha] T. Hahn (Ed.), International tables for crystallography, D. Reidel Publishing Company, (2005).

[He] J. Hempel, 3-manifolds, Princeton University Press, (1976).

[Hu] A. Hurwitz, Über algebraische Gebilde mit eindeutigen Transformationen in sich, Mathematische Annalen, 41(3) (1892), 403-442.

[HBLL+] S.T. Hyde, Z. Blum, T. Landh, S. Lidin, B.W. Ninham, S. Andersson and K. Larsson. The Language of Shape: The Role of Curvature in Condensed Matter. New York, USA: Elsevier, (1996).

[HOP] S.T. Hyde, M. O'Keeffe, D.M. Proserpio, A Short History of an Elusive Yet Ubiquitous Structure in Chemistry, Materials, and Mathematics, Angewandte Chemie Int. Ed. 47, (2008), 79968000.

[Ma1] C. L. May, Automorphisms of compact Klein surfaces with boundary, Pacific J. Math. 59 (1975), 199-210.

[Ma2] C. L. May, Large automorphism groups of compact Klein surfaces with boundary, Glasgow Math. J. 1 (1977), 1-10. 
[Me] W. H. Meeks, The conformal structure and geometry of triply periodic minimal surfaces in $R^{3}$, Bull. Amer. Math. Soc. 83 (1977), no. 1, 134-136.

[MY] W. H. Meeks, S. T. Yau, The equivariant Dehn's lemma and loop theorem, Commentarii Mathematici Helvetici, 56(1) (1981), 225-239.

[RCSR] M. O'Keeffe, M.A. Peskov, S.J. Ramsden, O.M. Yaghi, The Reticular Chemistry Structure Resource (RCSR) Database of, and Symbols for Crystal Nets Accts. Chem. Res. 41, (2008), 1782-1789.

[Schw] H. A. Schwarz, Gesammelte Mathematische Abhandlungen. Bd 1, Berlin: Springer, (1890).

[Scho] A. H. Schoen, NASA Tech. Note No. D-5541, (1970)

[WWZZ1] C. Wang, S. C. Wang, Y. M. Zhang, B. Zimmermann, Extending finite group actions on surfaces over $S^{3}$. Topology Appl. 160, (2013), no. 16, 2088-2103.

[WWZZ2] C. Wang, S. C. Wang, Y. M. Zhang, B. Zimmerman, Maximal Orders of Extendable Finite Group Actions on Surfaces. Groups Geom. Dyn. 9 (2015), no. 4, 1001-1045.

[WWZ] C. Wang, S. C. Wang, Y. M. Zhang, Maximal Orders of Extendable Actions on Surfaces. Acta Math. Sin. (Engl. Ser.) 32 (2016), no. 1, 54-68.

[Zi] B. Zimmermann, Uber Homomorphismen n-dimensionaler Henkelkrper und endliche Erweiterungen von Schottky-Gruppen, Comment. Math. Helv. 56 (1981), 474-486.

School of Mathematical Sciences, Peking University, Beijing 100871, CHinA

E-mail address: Bais@math.pku.edu.cn

Department of Applied Mathematics, Research School of Physics and Engineering, The Australian National University, Canberra, Australia

E-mail address: vanessa.robins@anu.edu.au

School of Mathematical Sciences, University of Science and Technology of China, Hefei 230026, CHINA

E-mail address: chao_wang_1987@126.com

School of Mathematical Sciences, Peking University, Beijing 100871, CHinA

E-mail address: wangsc@math.pku.edu.cn 